\title{
Versatile full Aperture Illumination OGSE Setup for Alignment and End-to-End Calibration of the EnMAP Hyperspectral Imager
}

M. Lettnera, T. Lamoura, L. Polza , W. Glastre ${ }^{b}$, E. Compain ${ }^{b}$, S. Fischer ${ }^{c}$

${ }^{a}$ OHB System AG, Manfred-Fuchs-Str. 1, D-82234 Weßling;

${ }^{b}$ Bertin Technologies, 155 rue Louis Armand, les Milles, 13290, Aix en Pce, France,

' DLR Space Administration, Königswinter Str. 522-524, 53227 Bonn, Germany

matthias.lettner@OHB.de

\section{Alignment and Calibration Requirements}

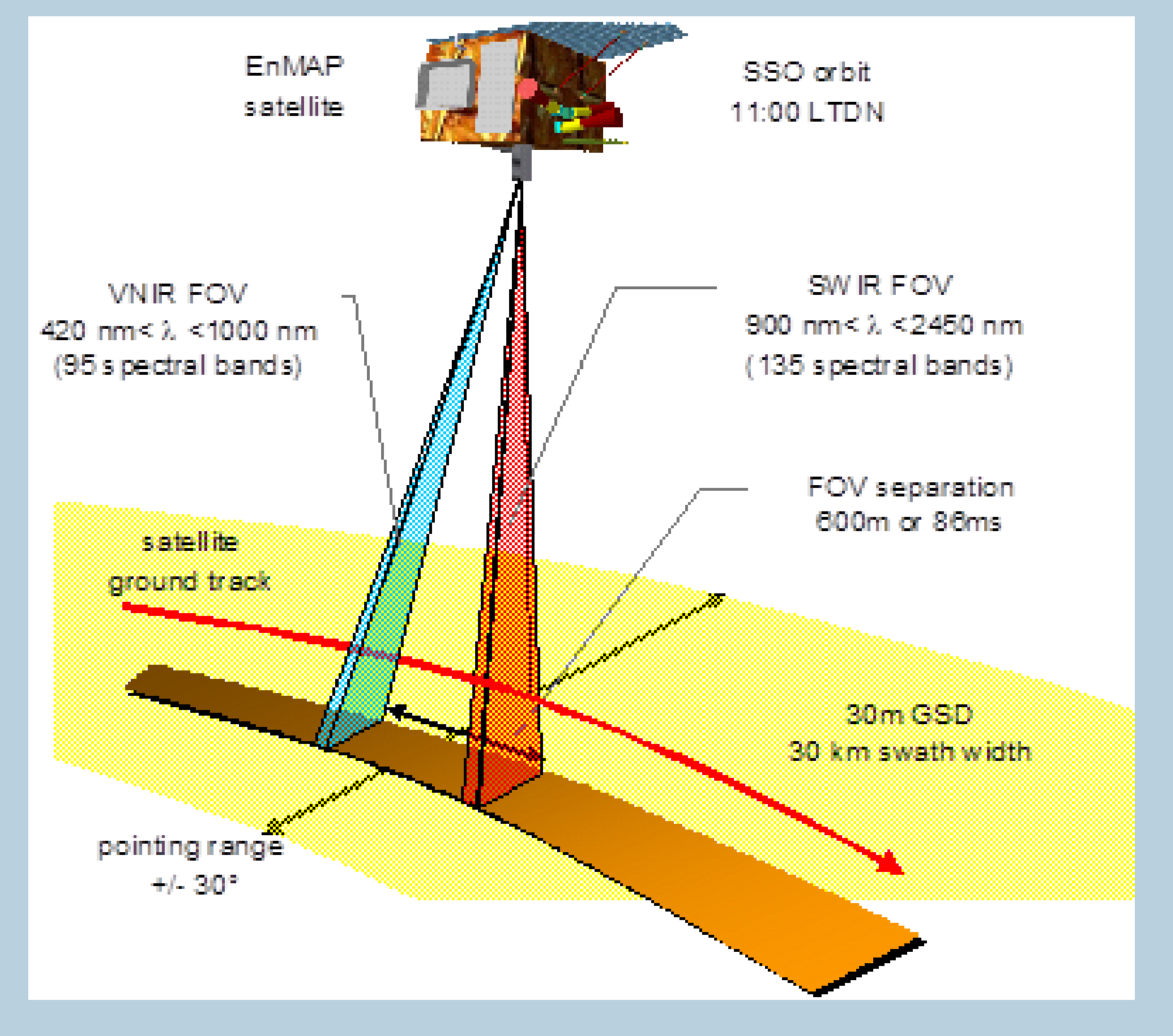

Telescope focussing

- Defocus $<\sim 10 \mu \mathrm{m}$

- For all field points

- For both channels

Spectral Calibration

- Pixel center wavelength

- Bandwidth

- Smile

- Spectral response function Spectral calibration acc. $<0.1$ SSD
Geometrical and Image quality calibration

- Line of sight of individual pixels

- Spectral channel co-registration

- Field-of-view (FOV)

- MTF

- Keystone

- Line spread function (LSF) across and along track

- Instrument/star sensors alignment

LoS measurement acc. $<1$ arcsec

\section{OGSE Development Approach}

Modular, multi-purpose Architecture

Maximize re-use and synergies of recurring tasks

- Multi purpose setup for efficient functional architecture \& AIT process

- Common multimode fiber Interface for light transport

- Separate light source and high performance optic

- Generic Interface allows to connect various light sources to various illumination systems

\section{OGSE Architecture}

\section{Wide range adjustable light source} (WIRAL)

- Monochromator based light source

- Wavelength range: $382 \mathrm{~nm}$ - $2481 \mathrm{~nm}$ gapless;

- Bandwidth $0.5 \mathrm{~nm}-2 \mathrm{~nm} \pm 3 \%$ (entire range);

- Absolute wavelength calibration and repeatability $<50 \mathrm{pm}$ (entire range);

- Smooth change of flux over Wavelength

- Radiometric

fluctuations $< \pm 0.5 \%$
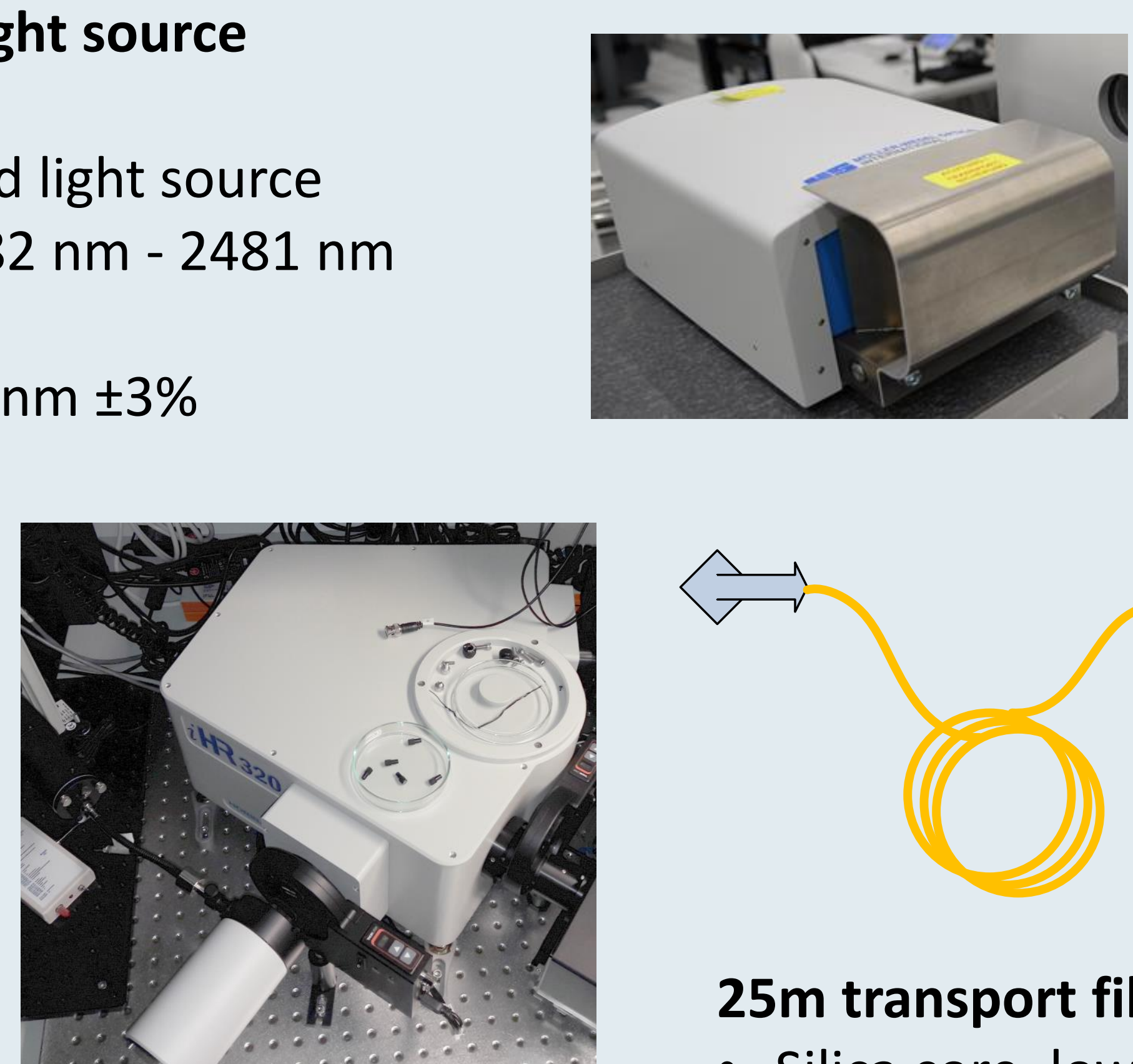

High dynamic range autocollimator (HDAC)

Electronc autocollimation telescope

- Development by Möller Wedel Optical

+/- $23 \mathrm{mrad}\left(1.32^{\circ}\right) \mathrm{x}+/-0,5 \mathrm{mrad}\left(0.03^{\circ}\right)$

- Accuracy (calibrated, to nat. st.) $1.2 \mu \mathrm{rad}$

- Light power $>0.3 \mu \mathrm{W}$ and $0.05 \mu \mathrm{W}$ for used bandwidth, coupled into

multimode fiber.

- White light mode

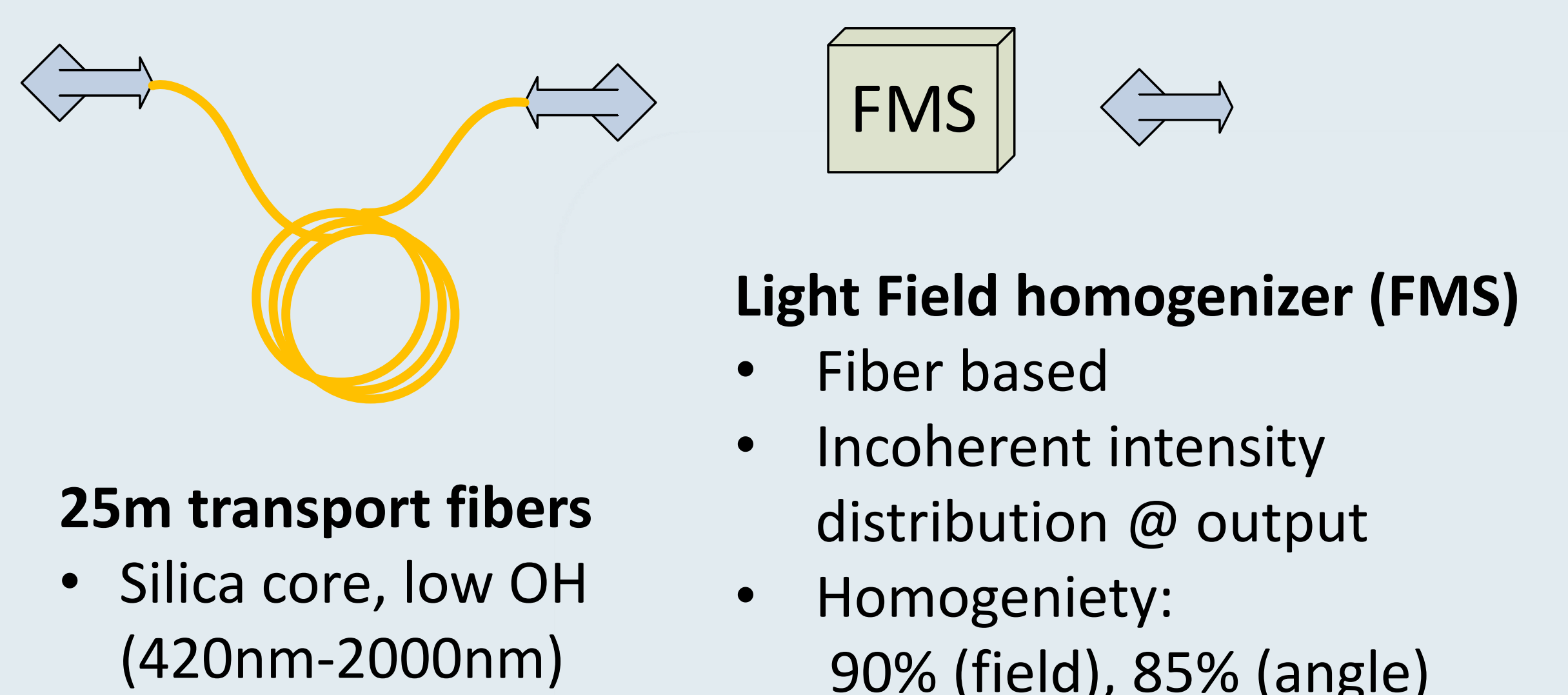

(420nm-2000nm)

ZRF core

90\% (field), 85\% (angle)

(2000nm - 2450nm)

- Control and telemetry via local and/or remote control

\section{WIRAL Performance}

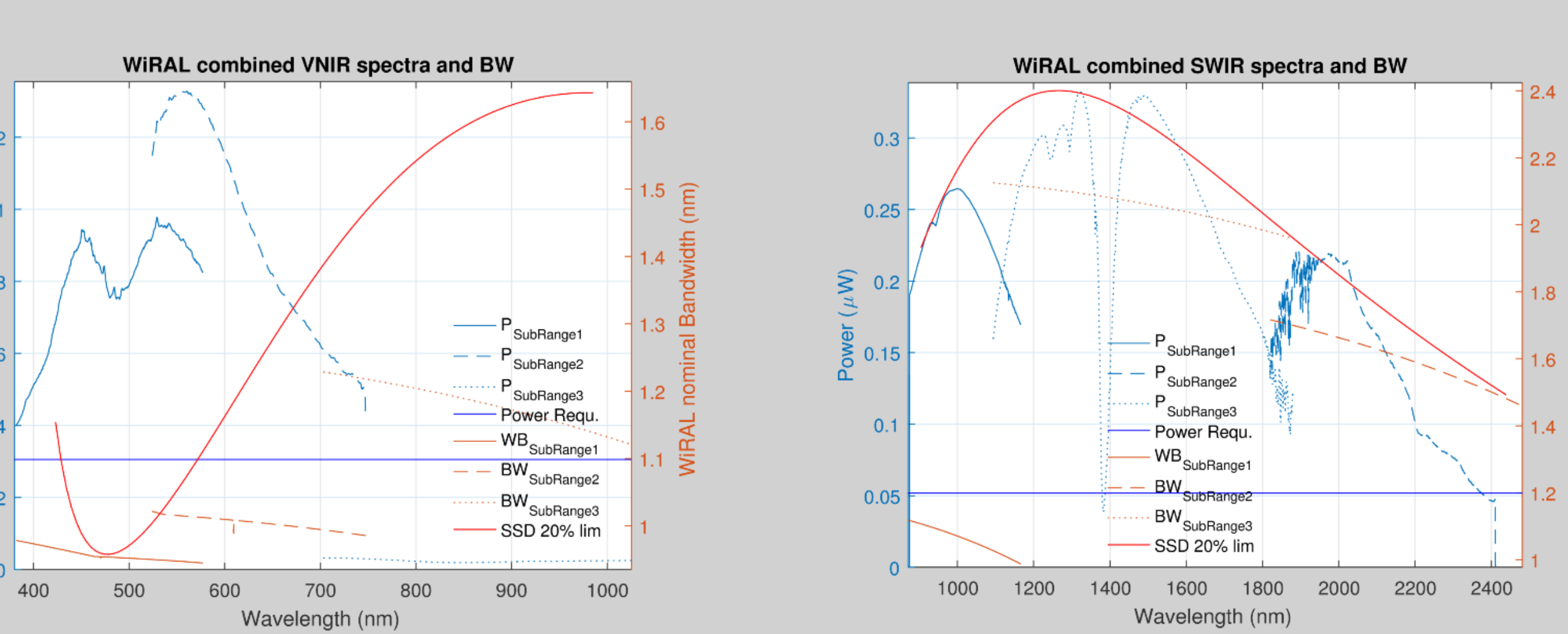

WIRAL Power and Bandwidth across entire wavelength range

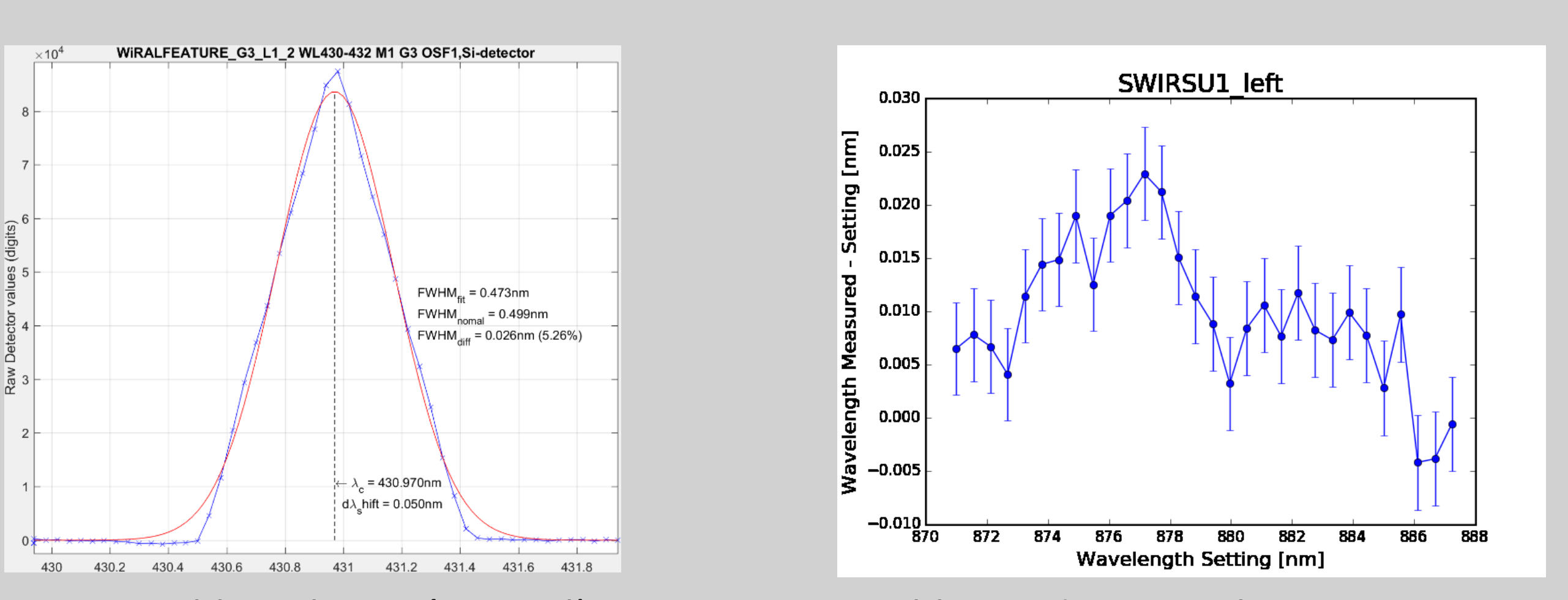

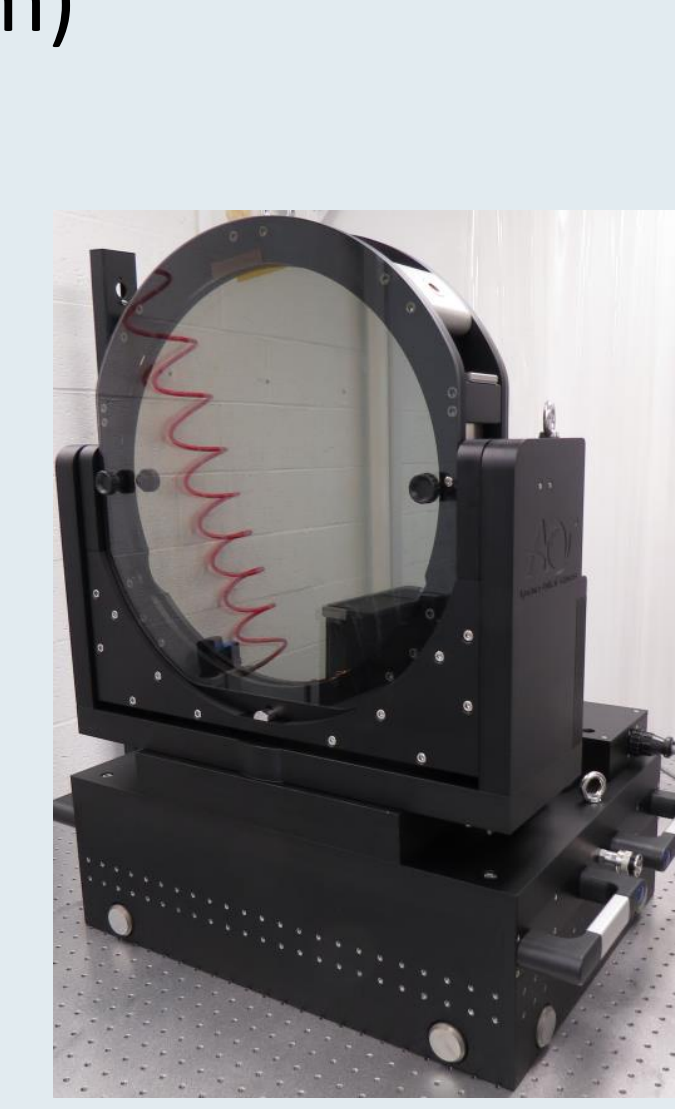

Full aperture Mirror (FAM)

- $330 \mathrm{~mm}$ flat reference Mirro

- SFE: $<6 n m$ (rms)

- Tip tilt gimble mount

- Stroke: $+/-2^{\circ}$

Incremental movement step: $<1 \mu \mathrm{rad}$

Floating movement on table using airpads

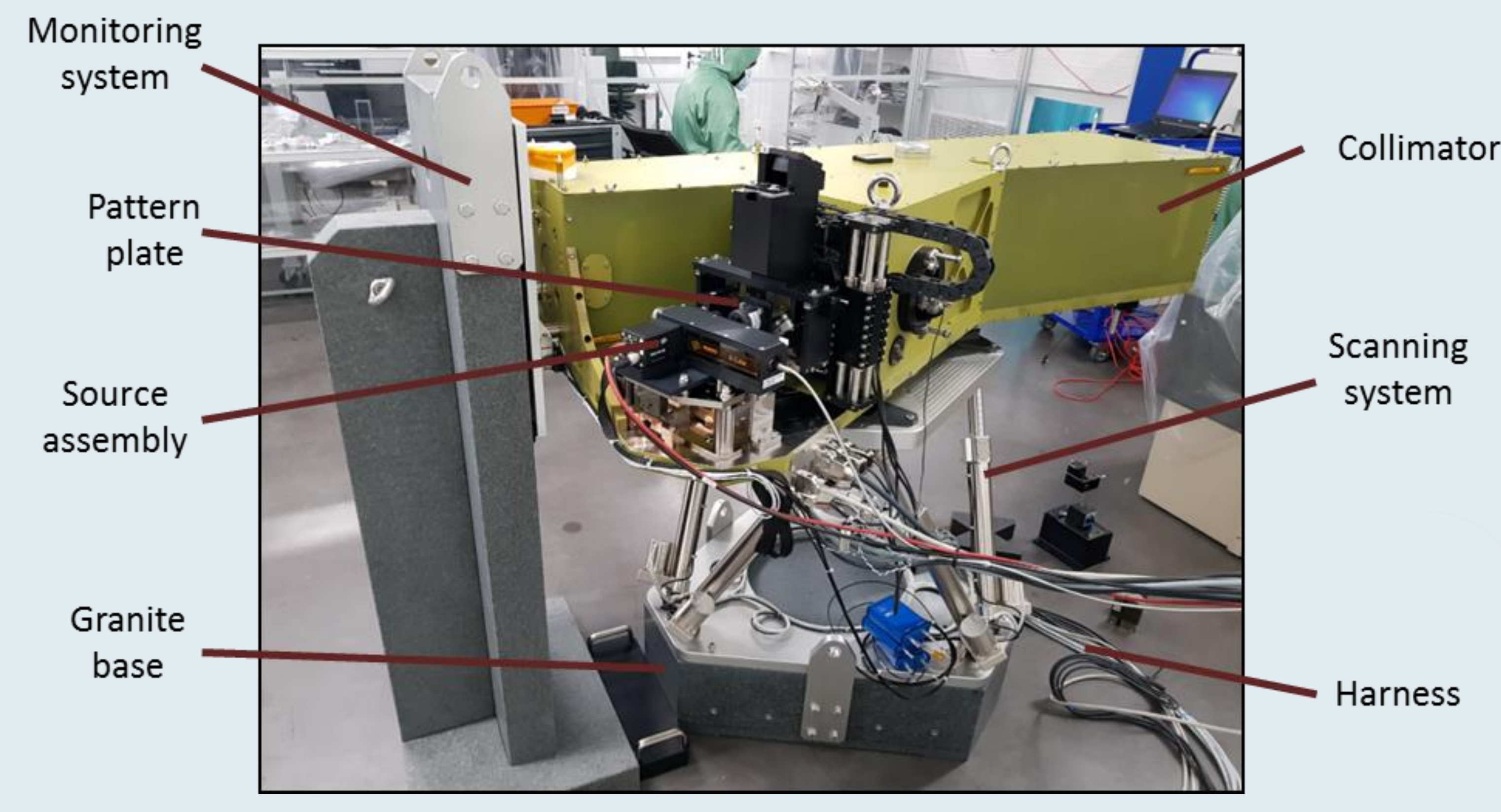

Full aperture illuminaton OGSE (FAI)

Design and manufacturing by Bertin technologies upon OHB Spec.

- 200mm unobscured 2-mirror collimator, field: 1.5x4arcmin

- Scene simulator (ALIO 2D stage and Optimask pattern plate)

- Source assembly including:

- Focus sensor \& 2-Mirror ocular with adjustable fiber Interface.

- Scanner/Positioning hexapod by Symetrie, to cover the HSI field of view.

- Angular stability monitoring system.

- Various configuratons to cover defocus, line of sight, MTF and spectral measurements

\section{FAl Performance}

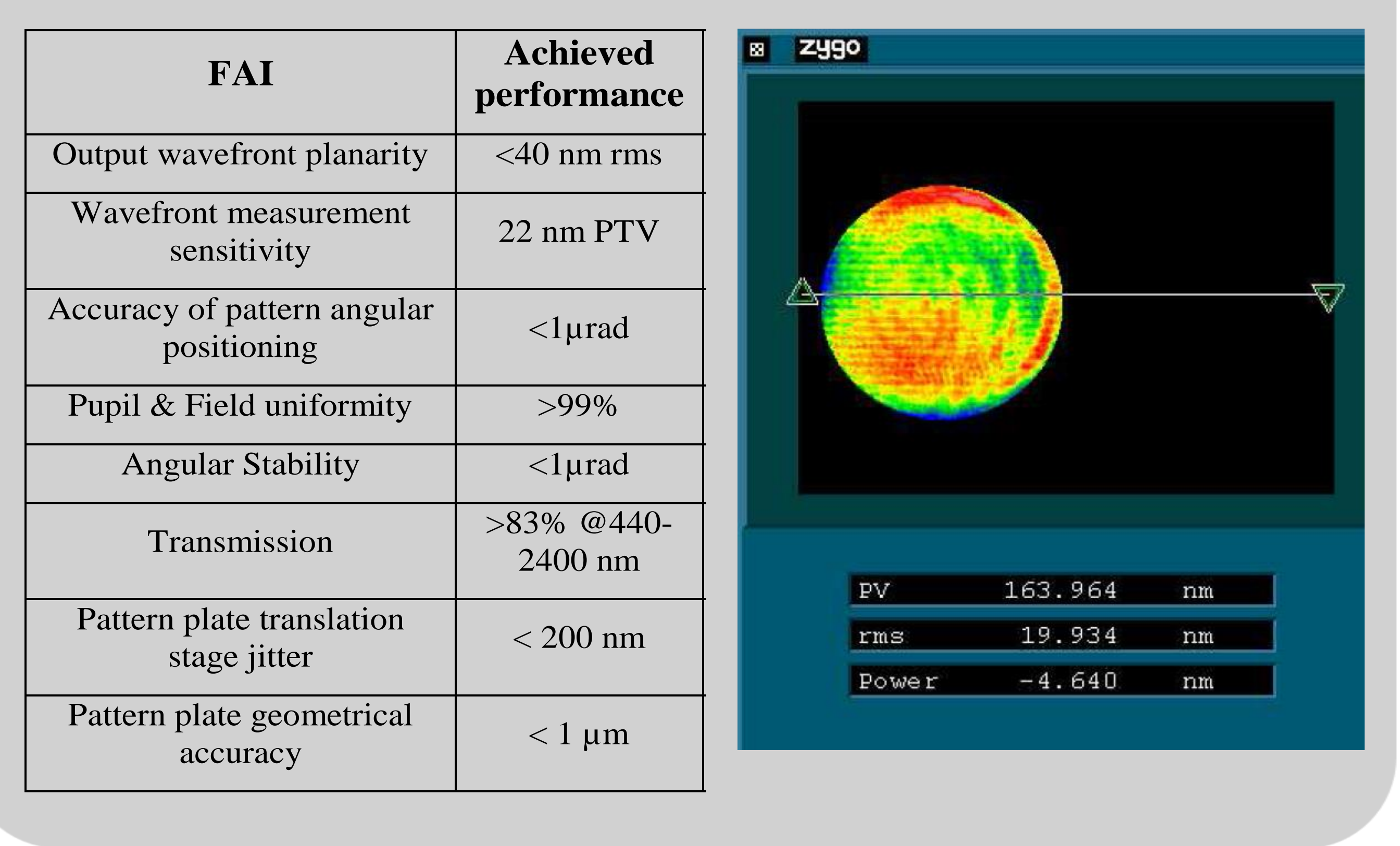

\section{LoS referencing}

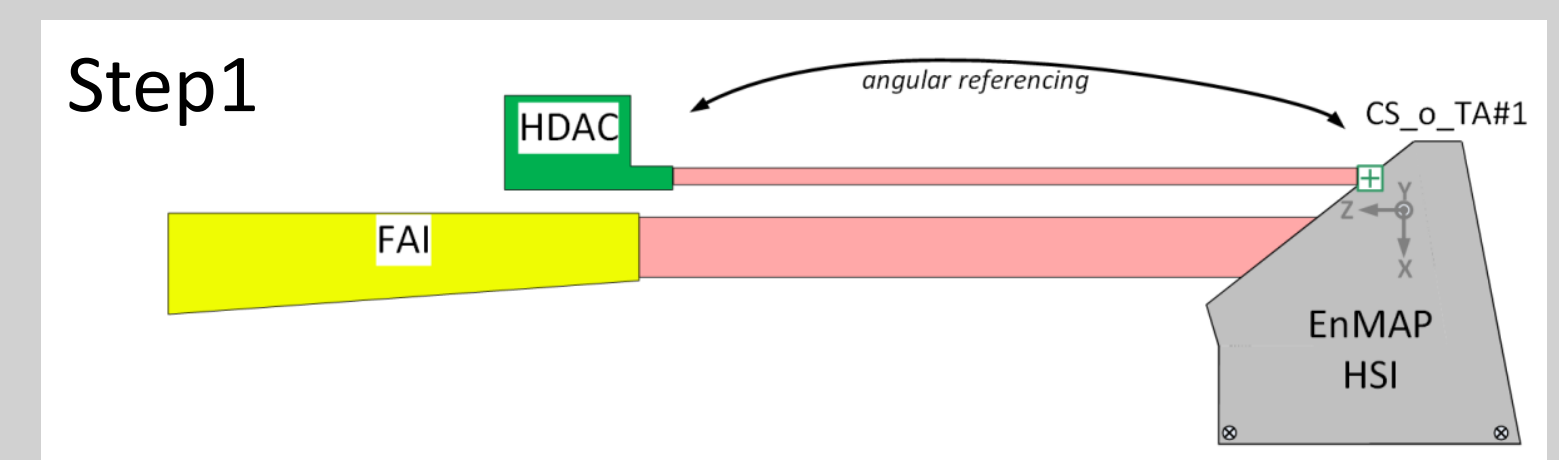

- Measure instrument reference frame with HDAC - Measure instrument LOS in FAI reference frame

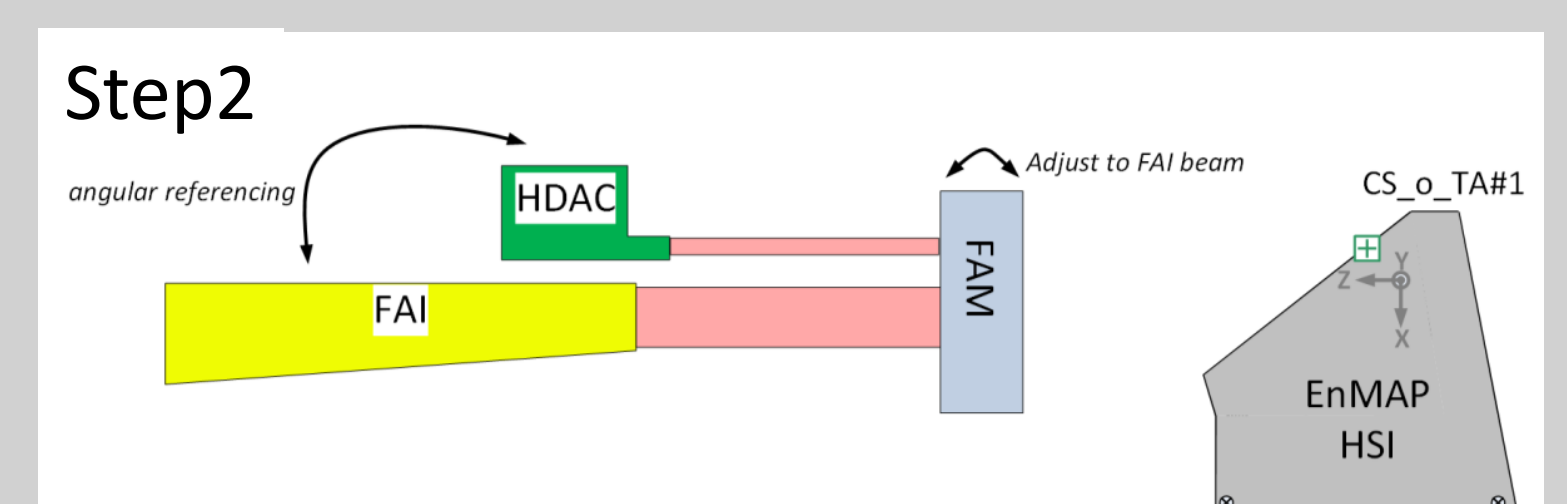

Place FAM between Instrument and OGSE Measure FAM orientation in FAl and HDAC reference frame 